\title{
Chondrocyte Apoptosis
}

\section{Implications for Osteochondral Allograft Transplantation}

\author{
Hubert T. Kim MD, PhD, Margie S. Teng BS, \\ Alexis C. Dang MD
}

Published online: 28 May 2008

(C) The Association of Bone and Joint Surgeons 2008

\begin{abstract}
Osteochondral allograft transplantation is a useful technique to manage larger articular cartilage injuries. One factor that may compromise the effectiveness of this procedure is chondrocyte cell death that occurs during the storage, preparation, and implantation of the osteochondral grafts. Loss of viable chondrocytes may negatively affect osteochondral edge integration and longterm function. A better understanding of the mechanisms responsible for chondrocyte loss could lead to interventions designed to decrease cell death and improve results. Recent studies indicate that apoptosis, or programmed cell death, is responsible for much of the chondrocyte death associated with osteochondral allograft transplantation. Theoretically, some of these cells can be rescued by blocking important apoptotic mediators. We review the role of apoptosis in cartilage degeneration, focusing on apoptosis associated
\end{abstract}

This research was performed at the San Francisco VA Medical Center.

One or more of the authors (HTK) has received funding from the Musculoskeletal Transplant Foundation, the Orthopaedic Research and Education Foundation, VA Medical Research, and the University of California, San Francisco.

Each author certifies that his or her institution has approved the animal protocol for this investigation and that all investigations were conducted in conformity with ethical principles of research.

\section{H. T. Kim (ه)}

Orthopedic Surgery Section, San Francisco VA Medical Center, 4150 Clement Street (112), San Francisco, CA 94121, USA

e-mail: kimh@orthosurg.ucsf.edu

M. S. Teng

Stanford University School of Medicine, Stanford, CA, USA

A. C. Dang

Department of Orthopedic Surgery, University of California, San

Francisco, San Francisco, CA, USA with osteochondral transplantation. We also review the pathways thought to be responsible for regulating chondrocyte apoptosis, as well as experiments testing inhibitors of the apoptotic pathway. These data suggest that key contributors to the apoptotic process can be manipulated to enhance chondrocyte survival. This knowledge may lead to better surgical outcomes for osteochondral transplantation.

\section{Introduction}

Osteochondral allograft transplantation has been shown to have good clinical results in short, intermediate, and longterm studies [21, 37, 48]. Osteochondral grafts can be supplied as frozen tissue, or as "fresh" or "cold-stored" grafts maintained in refrigerated media. The use of fresh and cold-stored grafts has the advantage of transplanting live chondrocytes that can survive long term and may contribute to the maintenance of the cartilage matrix after implantation [27, 36]. However, some of this potential advantage is lost during the storage and implantation of these grafts due to chondrocyte cell death [5]. Furthermore, iatrogenic cell death at the recipient site is likely to have an adverse effect on edge integration of the donor cartilage [50].

Recent reports support the theory that apoptosis, or programmed cell death (PCD), plays a major role in chondrocyte cell death under these conditions. To clarify the role of apoptosis in osteochondral allograft transplantation, we have performed a review of the scientific data related to the mechanisms and pathways responsible for chondrocyte apoptosis associated with this procedure. Furthermore, we have reviewed data suggesting that apoptosis inhibition can be an effective strategy to limit chondrocyte loss due to apoptosis. Theoretically, 
preservation of chondrocytes could enhance osteochondral graft integration and long-term function.

\section{Search and Selection Strategies}

We searched PubMed and Medline databases for peerreviewed literature using the search terms "osteochondral" and "allografts" (204 identified), "osteochondral" and "transplantation" (641 identified), and "chondrocyte" and "cartilage" and "apoptosis" (476 identified). The search was limited to journals available in English. Titles and abstracts were screened for relevance, and approximately 100 were selected for full review. In addition, we reviewed our own experimental data, both published and unpublished, in this area of research.

\section{Chondrocyte Apoptosis}

Cell death can occur through two distinct processes: necrosis and apoptosis. Necrosis is unregulated, ATPindependent, and results from catastrophic cellular damage. In contrast, apoptosis is a highly regulated process that is ATP-dependent and is triggered by stimuli that activate specific signaling and metabolic pathways [33, 49]. Apoptosis in normal adult articular cartilage is a rare event. However, a growing body of evidence suggests an "abnormal" increase in chondrocyte PCD is associated with cartilage degeneration in humans. Reports from Blanco et al. [6] and Hashimoto et al. [22] first established an association between increased levels of chondrocyte apoptosis and patients with osteoarthritis. While the actual percentage of cells undergoing apoptosis in these chronic forms of cartilage degeneration is debated [1], a majority of studies have reported mean chondrocyte apoptosis in the range of 1 to $6 \%[7,28,44]$. Much higher levels of chondrocyte apoptosis have been reported following intraarticular fractures in human retrieval studies. In the first of these reports, we analyzed cartilage specimens collected from patients undergoing surgery for tibial plateau fractures 0 to 14 days after injury [29]. The extent of chondrocyte apoptosis was measured using in situ DNA fragmentation labeling, commonly known as TUNEL (terminal deoxynucleotidyl transferase mediated dUTP Nick End Labeling) analysis. This assay is based upon specific labeling of short DNA fragments produced by inter-nucleosomal DNA cleavage that is a hallmark of true apoptosis. Independent confirmation of chondrocyte apoptosis was obtained through analysis of cell morphology and immunohistochemistry. On average, $18 \%$ of chondrocytes were TUNEL positive (range 1-44\%). Similarly, Murray and colleagues [38] reported an average of 35\% TUNEL positive cells in 30 retrieval specimens from various intraarticular fracture sites. In some specimens, the incidence of TUNEL positive cells exceeded $90 \%$.

\section{Chondrocyte Apoptosis Associated with Osteochondral} Graft Implantation

Osteochondral graft transplantation requires multiple physical manipulations of the donor graft that are prone to causing iatrogenic injury and apoptotic cell death. Borazjani and colleagues [8] recently reported that the repetitive impact necessary to insert human osteochondral grafts caused extensive cell death. Forty-eight hours after simulated graft insertion, $47 \% \pm 11 \%$ of cells in the superficial-most $0.5 \mathrm{~mm}$ of articular cartilage were dead compared to $7 \% \pm 4 \%$ in unloaded samples. After impaction, $26 \% \pm 7 \%$ of cells were TUNEL positive compared to $9 \% \pm 2 \%$ in controls. Furthermore, an increased numbers of cells staining positive for activated caspase 3 provided additional evidence that graft impaction was sufficient to cause substantial chondrocyte apoptosis.

Impaction is not the only manipulation likely to result in chondrocyte apoptosis. The initial step in osteochondral transplantation typically involves the creation of a cylindrical osteochondral defect to act as the recipient site for the osteochondral allograft. This defect is created with a motorized drill or similar coring instrument. Several studies have shown that drill injuries to articular cartilage cause extensive apoptosis in the surrounding cartilage $[12,15]$. Furthermore, this zone of injury extends outwards from the initial injury site over time [12]. As a result, a rim of relatively acellular matrix surrounds the recipient site. This apoptotic response has been hypothesized to impede effective cartilage integration in cartilage repair procedures [50].

Similarly, harvesting of the donor osteochondral graft from a larger piece of osteochondral tissue subjects the articular cartilage to mechanical damage and subsequent cell death. In most cases, a cylindrical osteotome or a powered trephine is used to harvest the graft(s). Huntley and colleagues [25] reported extensive chondrocyte death associated with human femoral osteochondral harvest using a cylindrical osteotome. These investigators reported a zone of cell death extending approximately $0.4 \mathrm{~mm}$ from the edge of the graft. Similar studies performed by Tew and colleagues [46] found that cell death due to this type of mechanical trauma was caused by a combination of necrotic and apoptotic cell death. Motorized coring devices are likely to cause even greater damage and cell death than manual trephines. This hypothesis was tested by Evans and colleagues [18] who reported that osteochondral grafts harvested with a powered trephine had approximately three 
times as much cell death as grafts harvested with a manual punch. Collectively, these studies demonstrate that the osteochondral transplantation procedure causes substantial chondrocyte apoptosis in the donor and recipient cartilage $[18,25,46]$.

\section{Chondrocyte Apoptosis Regulation Pathways}

Complex regulatory networks control the apoptotic process in all cells including chondrocytes (Fig. 1). The specific factors that promote chondrocyte apoptosis are not fully understood. However, it is likely matrix disruption plays a major role through the loss of integrin-mediated pro-survival signals $[9,10,24]$. Integrins are cell surface molecules that facilitate binding to other cells and to extracellular matrix (ECM) components. Binding to specific ECM components such as collagen mediates cell survival through downstream kinase-mediated signaling pathways. Loss of this pro-survival signal has been shown to induce apoptosis in cultured chondrocytes, while addition of the appropriate receptor ligand rescues the cells from apoptotic cell death $[9,10,24]$.

Another contributing factor is the release of pro-apoptotic cytokines, inflammatory mediators, and reactive

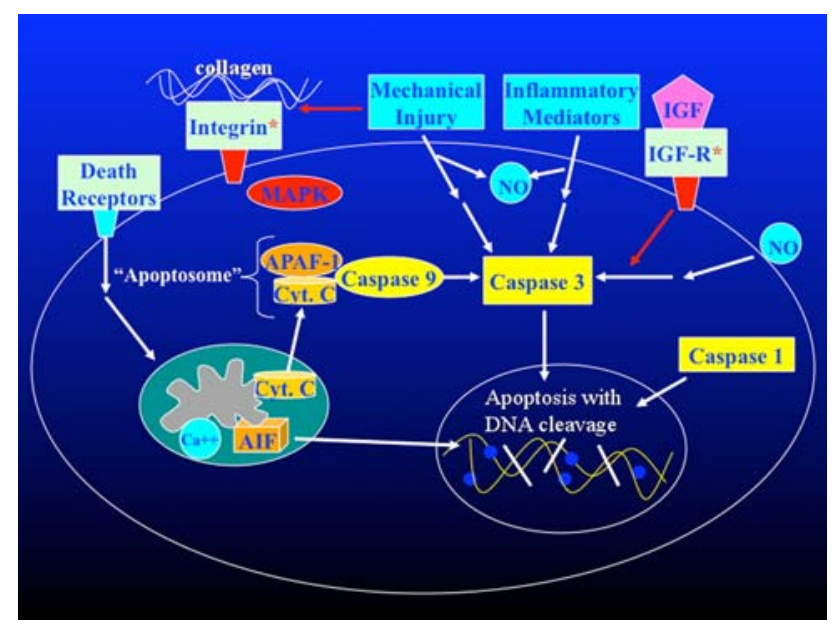

Fig. 1 Chondrocyte apoptotic pathways and mediators are shown in diagrammatic form. Integrin receptor and IGF receptor provide prosurvival signals. Ligand binding by so-called "death receptors" leads to translocation of cytochrome $\mathrm{c}$ from the mitochondria to the cytoplasm where it binds APAF-1 and caspase 9 to form an "apoptosome". The "apoptosome" is one of several activators of caspase 3, a central down-stream enzymatic mediator of the execution phase of apoptosis. Caspase 3 substrates include enzymes responsible for intranucleosomal DNA cleavage that is a hallmark of apoptosis. Mechanical injury, inflammatory mediators, and nitric oxide have been shown to cause chondrocyte apoptosis through caspase 3 dependent pathways. (AIF: apoptosis inducing factor; APAF-1: apoptotic protease activating factor-1 ;CytC: cytochrome C; IGF: insulin-like growth factor 1; IGF-R: insulin-like growth factor receptor; MAPK: mitogen activated protein kinase; NO: nitric oxide.) oxygen species by the surrounding tissues and infiltrating inflammatory cells [20]. For example, tumor necrosis factor (TNF)-alpha is a potent pro-apoptotic cytokine that binds to a specific cell surface "death receptor", triggering an apoptotic response in a wide range of cell types including chondrocytes [2]. The gaseous signaling molecule nitric oxide (NO) appears to be a dominant proapoptotic stimulus in articular cartilage [6, 23]. Recent studies have shown that chondrocyte apoptosis induced by shear forces and/or hydrostatic pressure is mediated in part by increased NO production [26,30]. Other reactive oxygen species such as hydrogen peroxide also induce apoptosis in cultured chondrocytes [3, 32].

Although a variety of different stimuli initiate chondrocyte apoptosis $[2,3,6,9,10,23,24,26,30,32]$, a universal feature of chondrocyte apoptosis is caspase activation [13, 16, 17, 31, 35, 39, 44] (Fig. 1). Caspases (cysteinyl aspartate-specific proteases) are key enzymatic mediators of PCD [33]. Upon activation, caspase enzymes cleave a number of cellular proteins, including poly (ADPribose) polymerase, sterol regulatory element binding protein, and nuclear mitotic apparatus proteins, which ultimately lead to cell death. In particular, caspase 1 and caspase 3 are believed to play critical roles in the initiation and execution phases of chondrocyte apoptosis, making them attractive targets for intervention [31]. Caspase 9, part of the "apoptosome" complex that mediates mitochondrial pro-apoptotic signaling pathways, may also play an important role in chondrocyte apoptosis [35].

\section{Inhibition of Chondrocyte Apoptosis In vitro}

Blockade of the pathways responsible for the initiation and/ or execution of apoptosis may prevent chondrocyte loss associated with cartilage injury, and a series of recent studies $[14,16,31,39]$ suggest this approach may, in fact, be quite feasible. Nuttall and colleagues [39] demonstrated that caspase 3 inhibition could block chondrocyte apoptosis and maintain biosynthetic function in cultured chondrocytes. Other studies $[14,16,31]$ have identified growth factors and multiple caspase inhibitors that can block chondrocyte PCD induced by matrix degradation and mechanical injury. We reported that insulin growth factor (IFG)-1, as well as peptide inhibitors of caspase 1 and caspase 3, blocked chondrocyte apoptosis induced by collagenases treatment [31]. Similarly, D'Lima and colleagues $[14,16]$ reported that IGF-1 and the non-specific caspase inhibitor ZVAD-fmk decrease apoptosis induced by injurious compression of cartilage explants.

Agents capable of modulating apoptosis are not limited to growth factors and caspase inhibitors. For example, the surfactant P188 has been shown to inhibit apoptosis in 
neuronal cells subjected to mechanical injury [43]. P188 is thought to modulate cell death by stabilizing damaged cell membranes. In a recent study, P188 was shown to decrease TUNEL positive chondrocytes by $45 \%$ following $25 \mathrm{MPa}$ of unconfined compression of bovine cartilage explants [4]. However, no other assays for apoptosis were employed in this study, thus precluding definitive conclusions about the effects of P188 on true apoptosis versus necrosis. Nitric oxide is another attractive target for apoptosis modulation. Inhibitors of nitric oxide synthetase have been shown to block apoptosis in articular cartilage explants isolated from animals with early osteoarthritis [41]. Collectively, this body of research suggests provide proof of concept for the use of apoptosis inhibitors to minimize chondrocyte apoptosis and to enhance the results of osteochondral allograft transplantation.

Inhibition of Chondrocyte Apoptosis After Cartilage Injury in vivo

The potential usefulness of apoptosis inhibition has also been demonstrated in animal models of degenerative arthritis. In a canine model, Pelletier and colleagues [40] demonstrated that selective inhibition of inducible nitric oxide synthetase reduced the progression of experimental osteoarthritis. This was associated with a reduction in chondrocyte apoptosis and expression of caspase 3. Similarly, D'Lima and colleagues [17] tested the hypothesis that intraarticular injection of caspase inhibitors would decrease the severity of osteoarthritis in a rabbit ACL transection model. These investigators found a reduction in the size and severity of cartilage lesions after treatment with the broad-spectrum caspase inhibitor ZVAD-fmk, a specific inhibitor of caspase 1, and the combination of caspase 3 and 8 specific inhibitors [17].

To establish the feasibility of apoptosis inhibition in the setting of acute injury in vivo, we tested the effects of a caspase inhibitor delivered directly into the knees of 10 rabbits after experimental osteochondral injuries [11]. Treated knees received daily intraarticular injections of ZVAD-fmk (100 $\mu$ M, 0.5 cc; Calbiochem, San Diego, CA, USA). Control knees received daily intraarticular injections of vehicle alone. The extent of PCD was quantified by TUNEL analysis and confirmed by anti-ssDNA antibody staining of heat-treated specimens, a highly specific test for true apoptosis [19]. Treatment with Z-VAD-fmk markedly reduced overall chondrocyte PCD as measured by TUNEL analysis (treated, $7.4 \% \pm 1.8 \%$; controls, $32.5 \% \pm 8.6 \%$; $\mathrm{p}<0.001)$. In areas greater than $1 \mathrm{~mm}$ from the injury site, PCD was reduced to essentially background levels $(1.2 \% \pm 1.6 \%)$.
Apoptosis Inhibition Enhances Chondrocyte Survival During Cold Storage of Osteochondral Grafts

A recent study found multiple key mediators of apoptosis are upregulated during cold storage of human osteochondral tissues [42]. Therefore, blockade of these mediators could enhance the survival of chondrocytes during storage and theoretically improve the results of osteochondral allograft transplantation surgeries. To test this theory, large osteochondral "grafts," each consisting of half of a bovine femoral hemicondyle, were placed in storage media (Dulbecco's modified eagle medium $\mathrm{H}-16$ ) at $4^{\circ} \mathrm{C}$. Half the grafts were stored in media alone and half were stored in media containing $50 \mu \mathrm{M}$ ZVAD-fmk to block caspase activity. At weekly intervals, multiple full-thickness articular cartilage specimens were harvested using a $4 \mathrm{~mm}$ biopsy punch. Four to eight punches per time point per condition were analyzed. The cylindrical specimens were halved, and half of each specimen was mounted on a sectioning block. The other half was fixed in zinc-buffered formalin for TUNEL analysis (see below). Unfixed samples were sectioned into $100-\mu \mathrm{m}$ slices using a vibratory microtome (Vibratome, St Louis, MO, USA).

Cell viability was assayed using the Live/Dead ( $\mathrm{R}$ Viability/Cytotoxicity Kit (Molecular Probes, OR, USA). Samples were incubated for 30 minutes at room temperature with a solution of $1 \mu \mathrm{mol}$ EthD- 1 and $1 \mu \mathrm{mol}$ calceinAM. These concentrations were modified from the manufacturer's protocol to optimize the sensitivity and specificity of the assay. Fluorescence microscopy images were captured with an Axiocam digital camera (Carl Zeiss, Thornwood, NY, USA) at a resolution of 1 megapixel. Cells were counted from a $1250-\mu \mathrm{m}$ wide by $1000-\mu \mathrm{m}$ deep area near the center of each slice. Semiautomated data collection and analysis were carried out using Adobe Photoshop (Adobe Systems Inc, San Jose, CA, USA) and a public domain Java image processing program (ImageJ; National Institutes of Health, Bethesda, MD, USA).

Specimens fixed in zinc-buffered formalin were paraffin embedded, and $7 \mu \mathrm{m}$ sections were prepared using a rotary microtome. TUNEL analysis was performed as previously described to identify cells with DNA fragmentation characteristic of cells undergoing apoptosis [13]. DAPI staining was used to verify that TUNEL positive cells exhibited nuclear morphology consistent with chondrocyte apoptosis.

After 3 weeks of storage, some TUNEL positive chondrocytes were identified throughout all layers of the cartilage (Fig. 2A). Not all TUNEL positive cells exhibited nuclear morphology consistent with apoptosis, suggesting that cell death was due to a combination of apoptosis and necrosis. Chondrocyte viability was significantly higher $(\mathrm{p}=0.005)$ with the addition of $50 \mu \mathrm{M}$ ZVAD-fmk to the storage media $(52.4 \% \pm 6.0 \%$ with $\mathrm{ZVAD}$-fmk versus 

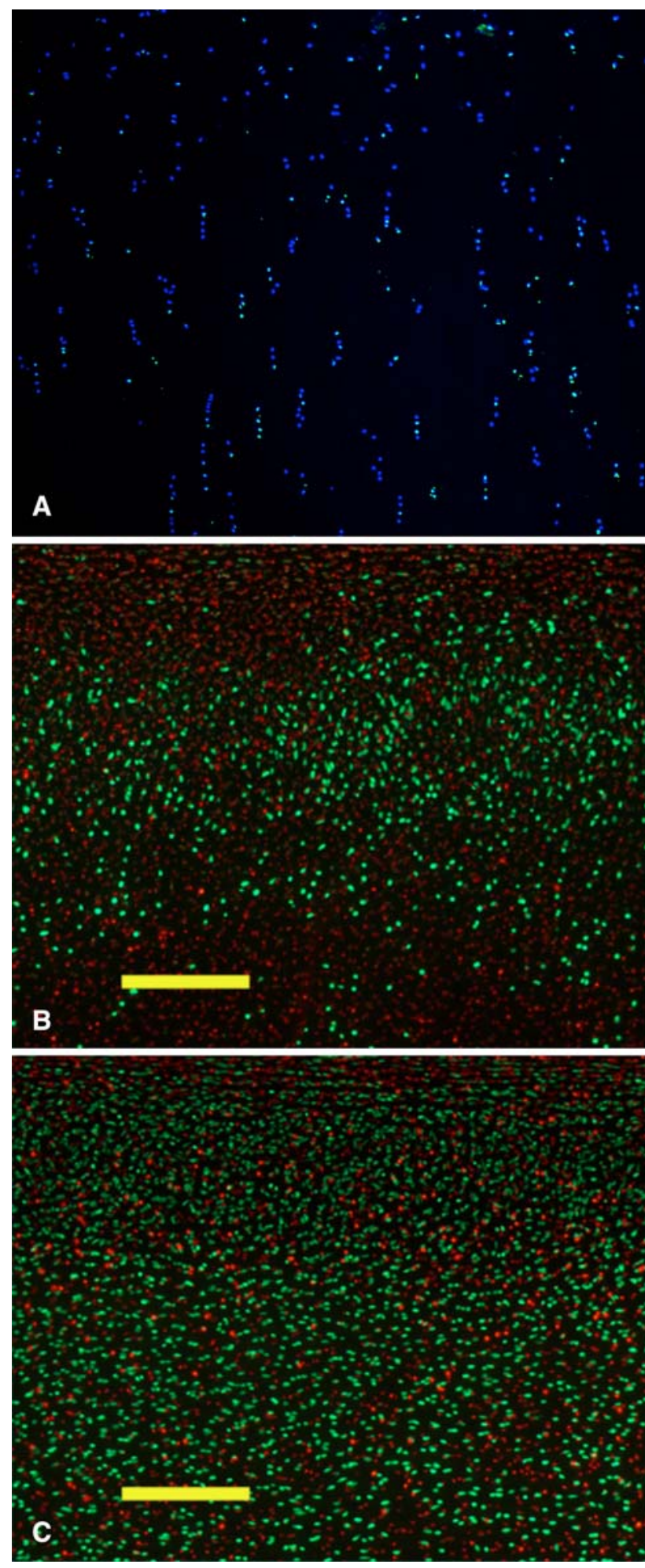

$29.6 \% \pm 4.9 \%$ control) (Fig. 2B-C). Importantly, the increased cellular viability was observed within the timeframe during which cold-stored human osteochondral grafts are most commonly used, between the second and fourth week of storage. These data suggest that apoptosis
4 Fig. 2A-C Chondrocyte apoptosis inhibition improves chondrocyte viability during cold storage of osteochondral grafts. Articular cartilage biopsy specimens after 3 weeks of storage at $4^{\circ} \mathrm{C}$ in DMEM were sectioned using a vibratory microtome. (A) TUNEL analysis demonstrates numerous positive (green) nuclei indicating DNA fragmentation that is a hallmark of apoptosis. DAPI (blue) counterstain shows nuclei of apoptotic and nonapoptotic cells. (B) Live/dead staining of biopsy specimens obtained from grafts stored in DMEM is shown. (C) DMEM supplemented with the caspase inhibitor ZVADfmk is shown. Live cells are stained green and dead cells are stained red. Scale bar $=250 \mu \mathrm{M}$.

inhibitors may be beneficial additives during cold storage of osteochondral grafts.

\section{Discussion}

A growing body of evidence supports the following hypotheses: (1) chondrocyte apoptosis is associated with cartilage injury and degeneration; (2) chondrocyte apoptosis is a consequence of osteochondral graft storage, preparation and implantation; and (3) chondrocyte apoptosis is at least partially preventable through the inhibition of key apoptotic pathways. Therefore, apoptosis inhibition would seem to be a rational strategy to pursue in order to enhance the clinical results of osteochondral allograft transplantation. Theoretically, apoptosis inhibition could increase the percentage of viable chondrocytes in cold-stored grafts, and extend the "shelf-life" of these grafts making them more available to surgeons. By limiting apoptosis induced by matrix damage localized to the peripheral margins of the donor and recipient sites, cartilage edge integration could be enhanced. Furthermore, by blocking apoptosis caused during graft impaction, chondrocyte viability, particularly viability of critical superficial zone cells, could enhance the function and durability of transplanted osteochondral grafts.

While the benefits of apoptosis inhibition have been demonstrated in animal models of degenerative arthritis, cartilage injury, and cartilage repair using marrow-stimulation techniques $[13,17,40]$, the in vivo effects of apoptosis inhibition in the setting of osteochondral allograft transplantation have not been reported. Based upon existing research, beneficial effects would be anticipated. Whether or not beneficial effects seen in animal models will translate into clinical efficacy remains an unanswered question. Furthermore, the difficulty in proving clinical efficacy should not be underestimated as demonstrated by the inability of recent randomized clinical trials to demonstrate differences between competing cartilage repair techniques such as microfracture, autologous chondrocyte implantation, and mosaicplasty [47]. Nevertheless, welldesigned animal studies are the obvious next step. 
Although small animal models can provide important information, studies in large animal models will be necessary since factors such as the thickness of articular cartilage and the size of transplanted grafts are likely to have a major effect on the extent and pattern of apoptosis that occurs and the response to apoptosis inhibition.

While it is generally thought that increased chondrocyte viability in transplanted osteochondral grafts will lead to superior long-term clinical results, this hypothesis has not been proven definitively. Frozen osteochondral grafts have been used successfully for decades, particularly in orthopaedic oncology. These grafts have no viable chondrocytes yet appear to function well clinically for many years. Mankin and colleagues [34] report on a large series of allograft reconstruction including frozen osteoarticular grafts, and the development of arthritis was stated to become a problem after approximately 6 years. However, only $16 \%$ of the patients in their series went on to joint replacement. Why the articular cartilage in these grafts does not degenerate more rapidly and consistently in the absence of viable cells is not well understood. We are not aware of any human studies directly comparing the results of osteochondral transplantation using fresh and frozen grafts. However, in a dog model, results using fresh grafts were shown to be markedly superior to frozen grafts [45], suggesting that cell viability is an important contributor to osteochondral graft performance.

From a practical standpoint, osteochondral allograft transplantation is a particularly attractive target for apoptosis inhibition therapy for several reasons. First, much of the treatment could be performed outside the body, greatly limiting systemic side effects. For example, very high doses of potentially toxic agents could be applied to the grafts ex vivo to maximize efficacy. Second, treatment could be initiated before mechanical injury when it is most effective rather than afterwards as would be the case for degenerative conditions and traumatic injuries. Third, treatment could be focused specifically during the intraoperative, or perhaps perioperative, period when apoptosis is maximal. In contrast, longer-term treatment would be necessary for degenerative conditions such as osteoarthritis. Fourth, since these procedures are almost always performed as an open procedure, treatment directed at the donor site could be focused specifically in that location, perhaps by direct application of a bioactive gel, rather than throughout the entire joint.

In summary, substantial scientific evidence demonstrates an important association of chondrocyte apoptosis with osteochondral allograft transplantation. In the not too distant future, this knowledge may be applied to design novel therapeutic approaches that may improve the clinical results of this surgical procedure.

\section{References}

1. Aigner T, Hemmel M, Neureiter D, Gebhard PM, Zeiler G, Kirchner T, McKenna L. Apoptotic cell death is not a widespread phenomenon in normal aging and osteoarthritis human articular knee cartilage: a study of proliferation, programmed cell death (apoptosis), and viability of chondrocytes in normal and osteoarthritic human knee cartilage. Arthritis Rheum. 2001;44:13041312.

2. Aizawa T, Kon T, Einhorn TA, Gerstenfeld LC. Induction of apoptosis in chondrocytes by tumor necrosis factor-alpha. J Orthop Res. 2001;19:785-96.

3. Asada S, Fukuda K, Nishisaka F, Matsukawa M, Hamanisi C. Hydrogen peroxide induces apoptosis of chondrocytes; involvement of calcium ion and extracellular signal-regulated protein kinase. Inflamm Res. 2001;50:19-23.

4. Baars DC, Rundell SA, Haut RC. Treatment with the non-ionic surfactant poloxamer P188 reduces DNA fragmentation in cells from bovine chondral explants exposed to injurious unconfined compression. Biomechan Model Mechanbiol. 2006;5:133-139.

5. Ball ST, Amiel D, Williams SK, Tontz W, Chen AC, Sah RL, Bugbee WD. The effects of storage on fresh human osteochondral allografts. Clin Orthop Relat Res. 2004;418:246-252.

6. Blanco FJ, Ochs RL, Schwarz H, Lotz M. Chondrocyte apoptosis induced by nitric oxide. Am J Pathol. 1995;146:75-85.

7. Blanco FJ, Guitian R, Vázquez-Martul E, de Toro FJ, Galdo F. Osteoarthritis chondrocytes die by apoptosis. A possible pathway for osteoarthritis pathology. Arthritis Rheum. 1998;41:284-289.

8. Borazjani BH, Chen AC, Bae WC, Patil S, Sah RL, Firestein GS, Bugbee WD. Effect of impact on chondrocyte viability during insertion of human osteochondral grafts. J Bone Joint Surg Am. 2006;88:1934-1943.

9. Cao L, Lee V, Adams ME, Kiani C, Zhang Y, Hu W, Yang BB. Beta-integrin-collagen interaction reduces chondrocyte apoptosis. Matrix Biol. 1999;18:343-355.

10. Cao L, Yang BB. Chondrocyte apoptosis induced by aggrecan G1 domain as a result of decreased cell adhesion. Exp Cell Res. 1999;246:527-537.

11. Costouros JG, Dang AC, Kim HT. Inhibition of chondrocyte apoptosis in vivo following osteochondral injury. Osteoarthritis Cartilage. 2003;11:756-759.

12. Costouros JG, Dang AC, Kim HT. Comparison of chondrocyte apoptosis in vivo and in vitro following acute osteochondral injury. J Orthop Res. 2004;22:678-683.

13. Dang AC, Warren A, Kim HT. Beneficial effects of intra-articular caspase inhibition therapy following osteochondral injury. Osteoarthritis Cartilage. 2006;14:526-532.

14. D'Lima DD, Hashimoto S, Chen PC, Colwell CW Jr, Lotz MK. Human chondrocyte apoptosis in response to mechanical injury. Osteoarthritis Cartilage. 2001;9:712-719.

15. D’Lima DD, Hashimoto S, Chen PC, Lotz MK, Colwell CW Jr. In vitro and in vivo models of cartilage injury. J Bone Joint Surg Am. 2001;83(Suppl 2 Pt 1):22-24.

16. D'Lima DD, Hashimoto S, Chen PC, Lotz MK, Colwell CW Jr. Prevention of chondrocyte apoptosis. J Bone Joint Surg Am. 2001;83(Suppl 2 Pt 1):25-26.

17. D'Lima D, Hermida J, Hashimoto S, Colwell C, Lotz M. Caspase inhibitors reduce severity of cartilage lesions in experimental osteoarthritis. Arthritis Rheum. 2006;54:1814-1821.

18. Evans PJ, Miniaci A, Hurtig MB. Manual punch versus power harvesting of osteochondral grafts. Arthroscopy. 2004;20:306310.

19. Frankfurt OS, Krishan A. Identification of apoptotic cells by formamide-induced DNA denaturation in condensed chromatin. J Histochem Cytochem. 2001;49:369-378. 
20. Green DM, Noble PC, Ahuero JS, Birdsall HH. Cellular events leading to chondrocyte death after cartilage impact injury. Arthritis Rheum. 2006;54:1509-1517.

21. Gross AE, Shasha N, Aubin P. Long-term followup of the use of fresh osteochondral allografts for posttraumatic knee defects. Clin Orthop Relat Res. 2005;435:79-87.

22. Hashimoto S, Ochs RL, Komiya S, Lotz M. Linkage of chondrocyte apoptosis and cartilage degradation in human osteoarthritis. Arthritis Rheum. 1998;41:1632-1638.

23. Hashimoto S, Takahashi K, Amiel D, Coutts RD, Lotz M. Chondrocyte apoptosis and nitric oxide production during experimentally induced osteoarthritis. Arthritis Rheum. 1998;417:1266-1274.

24. Hirsch MS, Lunsford LE, Trinkaus-Randall V, Svoboda KK. Chondrocyte survival and differentiation in situ are integrin mediated. Dev Dyn. 1997;210:249-263.

25. Huntley JS, Bush PG, McBernie JM, Simpson AH, Hall AC. Chondrocyte death associate with femoral osteochondral harvest as performed for mosaicplasty. $J$ Bone Joint Surg Am. 2005;87:351-360.

26. Islam N, Haqqi TM, Jepsen KJ, Kraay M, Welter JF, Goldberg VM, Malemud CJ. Hydrostatic pressure induces apoptosis in human chondrocytes from osteoarthritic cartilage through upregulation of tumor necrosis factor-alpha, inducible nitric oxide synthase, p53, c-myc, and bax-alpha, and suppression of bcl-2. J Cell Biochem. 2002;87:266-278.

27. Jamali AA, Hatcher SL, You Z. Donor cell survival in a fresh osteochondral allograft at twenty-nine years. A case report. J Bone Joint Surg Am. 2007;89:166-169.

28. Kim HA, Lee YJ, Seong SC, Choe KW, Song YW. Apoptotic chondrocyte death in human osteoarthritis. $J$ Rheumatol. 2000;27:455-462.

29. Kim HT, Lo M, Pillarisetty R. Chondrocyte apoptosis following intra-articular fracture in humans. Osteoarthritis Cartilage. 2002;10:747-749.

30. Lee MS, Trindade MC, Ikenoue T, Goodman SB, Schurman DJ, Smith RL. Regulation of nitric oxide and bcl-2 expression by shear stress in human osteoarthritic chondrocytes in vitro. $J$ Cell Biochem. 2003;90:80-86.

31. Lo MY, Kim HT. Chondrocyte apoptosis induced by collagen degradation: inhibition by caspase inhibitors and IGF-1. J Orthop Res. 2004;22:140-144.

32. Lo MY, Kim HT. Chondrocyte apoptosis induced by hydrogen peroxide requires caspase activation but not mitochondrial pore transition. J Orthop Res. 2004;22:1120-1125.

33. Majno G, Joris I. Apoptosis, oncosis, and necrosis. An overview of cell death. Am J Pathol. 1995;146:3-15.

34. Mankin HJ, Gebhardt MC, Jennings LC, Springfield DS, Tomford WW. Long-term results of allograft replacement in the management of bone tumors. Clin Orthop Relat Res. 1996; 324:86-97.

35. Matsuo M., Nishida K, Yoshida A, Murakami T, Inoue $H$. Expression of caspase-3 and -9 relevant to cartilage destruction and chondrocyte apoptosis in human osteoarthritic cartilage. Acta Med Okayama. 2001;55:333-340.
36. Maury AC, Safir O, Heras FL, Pritzker KP, Gross AE. Twentyfive-year chondrocyte viability in fresh osteochondral allograft. A case report. J Bone Joint Surg Am. 2007;89:159-165.

37. McCulloch PC, Kang RW, Sobhy MH, Hayden JK, Cole BJ. Prospective evaluation of prolonged fresh osteochondral allograft transplantation of the femoral condyle: minimum 2-year followup. Am J Sports Med. 2007;35:411-420.

38. Murray MM, Zurakowski D, Vrahas MS. The death of articular chondrocytes after intra-articular fracture in humans. J Trauma. 2004;56:128-131.

39. Nuttall ME, Nadeau DP, Fisher PW, Wang F, Keller PM, DeWolf WE Jr, Goldring MB, Badger AM, Lee D, Levy MA, Gowen M, Lark MW. Inhibition of caspase-3-like activity prevents apoptosis while retaining functionality of human chondrocytes in vitro. J Orthop Res. 2000;18:356-363.

40. Pelletier JP, Jovanovic DV, Lascau-Coman V, Fernandes JC, Manning PT, Connor JR, Currie MG, Martel-Pelletier J. Selective inhibition of inducible nitric oxide synthase reduces progression of experimental osteoarthritis in vivo: possible link with the reduction in chondrocyte apoptosis and caspase 3 level. Arthritis Rheum. 2000;43:1290-1299.

41. Pelletier JP, Fernandes JC, Jovanovic DV, Reboul P, MartelPelletier J. Chondrocyte death in experimental osteoarthritis is mediated by MEK $1 / 2$ and p38 pathways: role of cyclooxygenase-2 and inducible nitric oxide synthase. $J$ Rheumatol. 2001;28:2509-2519.

42. Robertson CM, Allen RT, Pennock AT, Bugbee WD, Amiel D. Upregulation of apoptotic and matrix-related gene expression during fresh osteochondral allograft storage. Clin Orthop Relat Res. 2006;442:260-266.

43. Serbest G, Horwitz J, Jost M, Barbee K. Mechanisms of cell death and neuroprotection by poloxamer 188 after mechanical trauma. FASEB J. 2006;20:308-310.

44. Sharif M, Whitehouse A, Sharman P, Perry M, Adams M. Increased apoptosis in human osteoarthritic cartilage corresponds to reduced cell density and expression of caspase-3. Arthritis Rheum. 2004;50:507-515.

45. Stevenson S, Dannucci GA, Sharkey NA, Pool RR. The fate of articular cartilage after transplantation of fresh and cryopreserved tissue-antigen-matched and mismatched osteochondral allografts in dogs. J Bone Joint Surg Am. 1989;71:1297-1307.

46. Tew SR, Kwan AP, Hann A, Thomson BM, Arcerh CW. The reactions of articular cartilage to experimental wounding: role of apoptosis. Arthritis Rheum. 2000;43:215-225.

47. Wasiak J, Clar C, Villanueva E. Autologous cartilage implantation for full thickness articular cartilage defects of the knee. Cochrane Database Syst Rev. 2006;3:CD005489.

48. Williams RJ 3rd, Ranawat AS, Potter HG, Carter T, Warren RF. Fresh stored allografts for the treatment of osteochondral defects of the knee. J Bone Joint Surg Am. 2007;89:718-726.

49. Wyllie AH, Kerr JF, Currie AR. Cell death: the significance of apoptosis. Int Rev Cytol. 1980;68:251-306.

50. Zhang Z, McCaffery JM, Spencer RGS, Francomano CA. Growth and integration of neocartilage with native cartilage in vitor. J Orthop Res. 2005;23:433-439. 